\title{
Effect of Replacing the Aspartic Acid/Glutamic Acid Residues of Bullfrog Sialic Acid Binding Lectin with Asparagine/Glutamine and Arginine on the Inhibition of Cell Proliferation in Murine Leukemia P388 Cells
}

\author{
Yuko Ogawa, ${ }^{a}$ Masanori Iwama, ${ }^{*, a, 1)}$ Kazuko OhgI,${ }^{a}$ Tsutomu TsujI,${ }^{a}$ Masachika IrIE, ${ }^{a}$ \\ Tadashi ITAGaki, ${ }^{b}$ Hiroko KoBaYAshi, ${ }^{b}$ and Norio INOKUCHI ${ }^{b}$ \\ ${ }^{a}$ Department of Microbiology, Hoshi College of Pharmacy; 2-4-41 Ebara, Shinagawa-ku, Tokyo 142-8501, Japan: and \\ ${ }^{b}$ Department of Microbiology, College of Pharmacy, Nihon University; Narashinodai, Funabashi, Chiba 274-8555, Japan. \\ Received December 11, 2001; accepted March 1, 2002
}

\begin{abstract}
The sialic acid binding lectin from bullfrog oocytes (cSBL) is known to have anti-tumor activity. In a previous report, to elucidate the relationship between the net charge and anti-tumor activity of cSBL, we examined the effect of chemical modifications of cSBL with a water-soluble carbodiimide in the presence of various nucleophiles. The results suggested that the anti-tumor activity and internalization into tumor cells increased with an increase in the net charge of cSBL. However, in the chemically modified cSBL, a modification site was observed on average in two of the carboxyl groups of cSBL. To confirm these previous results and to determine which modified carboxyl group contributes to the increase in anti-tumor activity, we prepared mutants with substitutions of Asn/Gln and Arg at three acidic amino acid residues of cSBL and studied their anti-tumor activity and internalization efficiency. The results showed the enhancing effect of charge on anti-tumor activity and internalization, and suggested that the replacement of D24 and E88 of cSBL with arginine is more effective than that of E97. The double mutant D24RE88R showed comparable anti-tumor activity to the ethylenediamine-modified cSBL reported previously. The mutant was well-characterized as a pure cSBL derivative suitable for studying the mechanism of the anti-tumor action of cSBL.
\end{abstract}

Key words sialic acid binding lectin; ribonuclease; anti-tumor; site-directed mutagenesis

Sialic acid binding lectin from Rana catesbeiana oocyte (cSBL) is known to have anti-tumor activity. ${ }^{2)} \mathrm{cSBL}$ is a multifunctional protein and is recognized as a pyrimidine basespecific ribonuclease. ${ }^{3)}$ In respect to its ribonuclease activity, cSBL belongs to the RNase A superfamily, ${ }^{4,5)}$ and is a very heat-stable enzyme. It retains $100 \%$ activity after heating at $100{ }^{\circ} \mathrm{C}$ for $\left.5 \mathrm{~min} .{ }^{6}\right)$ Four RNases have been isolated from the Rana family of frogs, and their primary structures have been determined to date. ${ }^{7-10)}$ All have a very similar primary structure, as shown in Fig. 1. Each of these RNases has antitumor activity, except the one from bullfrog liver. ${ }^{5)}$ Among them, the RNase from $R$. pipiens (onconase) has been studied most extensively and is in phase III clinical trials as an antitumor drug. ${ }^{11)}$ Nitta et al. reported on the anti-tumor activity of cSBL. ${ }^{2}$ The mechanism of the anti-tumor action of frog oocyte RNases was studied using cSBL and onconase, but has not yet been fully elucidated. Nitta et al. proposed that cSBL interacts with a sialoglycoprotein on the surface of tumor cells, is then internalized and works in the cells. ${ }^{5)}$ The proposal was based on the fact that cSBL was not effective against murine leukemia P388 cells treated with sialidase. On the other hand, $\mathrm{Wu}, \mathrm{N}$. Y. reported (cited in ref. 11) that the anti-tumor activity of onconase is not affected by pretreatment with sialidase, in spite of its structural similarity with cSBL.

Nitta et al. reported that the chemical modification of cSBL with a water soluble carbodiimide (EDC) in the presence of glycine methylester increased its anti-tumor action. ${ }^{2}$ In the previous paper, ${ }^{12}$ ) we reported that (i) modification of the carboxyl groups of cSBL with EDC in the presence of nucleophiles enhanced the cytotoxic activity toward P388 cells. The enhancing effect is dependent on the increase in positive net charge, thus the cytotoxicity was greatest in the order of cSBL modified in the presence of ethylenediamine $($ EDC-ED cSBL) $>$ glycine methylester (EDC-GM cSBL) $>$ taurine (EDC-TA cSBL). (ii) cSBL with a higher net charge is more efficiently internalized into the P388 cells than the native cSBL. However, we have not been able to define which carboxyl group is chemically modified in each sample. (iii) EDC-ED cSBL hydrolyzed RNA hybridized with RNA or DNA more effectively than the native cSBL. (iv) The base specificity especially that of the B2 site of EDC-ED cSBL, was altered by the chemical modification. ${ }^{12,13)}$

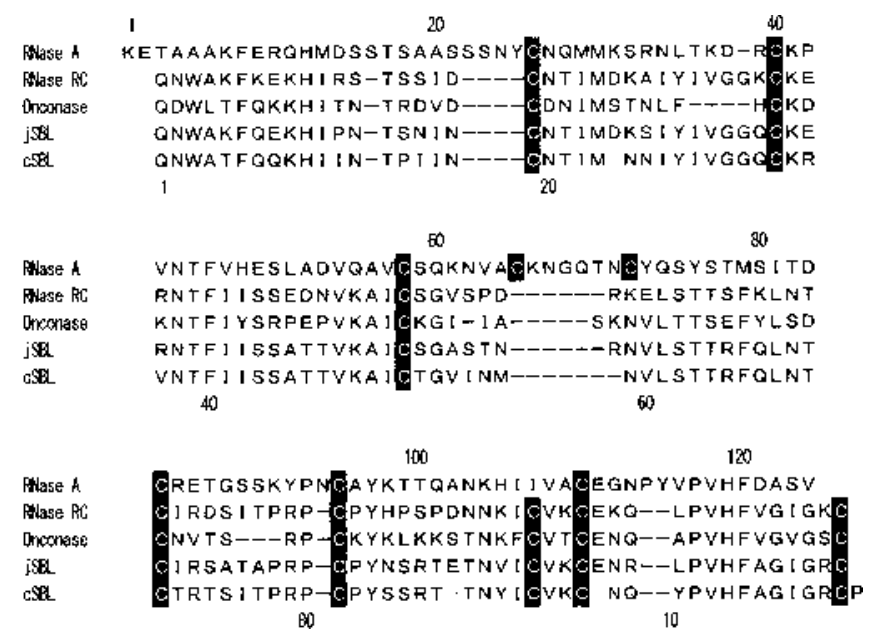

Fig. 1. Amino Acid Sequences of Four Frog RNases and Bovine Pancreatic RNase A

RNase A, bovine pancratic RNase $\mathrm{A}^{4}$; RNase RC, RNase from bullfrog liver ${ }^{7}$; Onconase, RNase from leopard frog $^{8}$; $;$ jSBL, sialic acid binding lection from the oocyte of Japanese frog ${ }^{9}$; cSBL, sialic acid binding lectin from the oocyte of bullfrog. ${ }^{10)} \mathrm{Cys}$ residues are written in white letters on a black ground, and the targets for mutations are written in bold. 
In this report, to clarify which amino acid residues of cSBL when modified have a marked effect on the anti-tumor activity, we prepared mutant cSBLs in which the acidic amino acid residues (aspartic acid or glutamic acid) were replaced with the corresponding acidic amides (asparagine or glutamine) or arginine, and studied their anti-tumor activity and enzymatic properties.

\section{MATERIALS AND METHODS}

Enzymes cSBL was purified according to the method of Nitta et $a l .^{2)}$

Cells Murine leukemia P388 cells and human leukemia HL60 cells were obtained from Health Science Research Resources Bank (Osaka, Japan).

Reagents RPMI1640 medium, ASF104 medium and bovine fetal serum were obtained from Invitrogen Corp. (Carlsbad, CA, U.S.A.), Ajinomoto (Tokyo, Japan) and Intergen (Purchase, NY, U.S.A.), respectively. Yeast RNA was a product of Marine Bio (Tokyo, Japan). The Mono S column and Heparin Sepharose CL6B were obtained from Amersham Pharmacia Biotech, AB (Uppsala, Sweden). Phosphocellulose, P11, was obtained from Watman Japan (Tokyo, Japan). Poly-L-lysine was obtained from Sigma (St. Louis, MO, U.S.A.).

Assay for RNase Activity (a) RNase activity toward RNA: RNase activity was measured according to a previous method $^{6)}$ with RNA $(0.25 \mathrm{mg} / \mathrm{ml})$ as a substrate in $50 \mathrm{~mm}$ Tris- $\mathrm{HCl}$ buffer ( $\mathrm{pH} 7.5$ ) at $37^{\circ} \mathrm{C}$. One unit of enzyme was defined as the amount which increased absorbance by 1.0 at $260 \mathrm{~nm}$ in $5 \mathrm{~min}$ under the experimental conditions. (b) Hydrolysis of poly $\mathrm{U}$ - poly A was measured spectrophotometrically in $25 \mathrm{~mm}$ Tris- $\mathrm{HCl}$ buffer (pH 7.5) containing $125 \mathrm{~mm}$ $\mathrm{NaCl}$ at $25^{\circ} \mathrm{C}$ as described previously. ${ }^{13)}$

Protein Concentration The concentration of cSBL was measured spectrophotometrically taking the absorbance at $280 \mathrm{~nm}$ of a $0.1 \%$ solution to be 1.0 .

Measurement of Circular Dichroism (CD) Spectrum The CD spectrum of the cSBL solution was measured with a J-600 spectropolarimeter (JASCO, Tokyo, Japan).

Anti-cell Proliferative Activity of cSBL Anti-cell proliferative activity was measured with murine leukemia P388 cells or human leukemia HL60 cells, according to the method of Nitta et al. ${ }^{2)}$ P388 cells were cultured in RPMI1640 supplemented with $10 \%$ fetal calf serum. The cells were collected by centrifugation, then suspended in the medium and diluted to $2 \times 10^{5}$ cells $/ \mathrm{ml}$. In a 96-well plate, with each well containing $200 \mu \mathrm{l}$ of the cell suspension, $10 \mu \mathrm{l}$ of various concentrations of cSBL or mutant cSBL previously filtered with a Millipore filter (Millex-GV) was added. Viable cells were counted after $48 \mathrm{~h}$ incubation at $37^{\circ} \mathrm{C}$ under $5 \% \mathrm{CO}_{2}$. Inhibition activity of cell proliferation in P388 cells was calculated as the percentage decrease of the increase in cell number with cSBL against the increase in the control cell number without cSBL. The $\mathrm{IC}_{50}$ of human leukemia HL60 cells was measured by the same method.

Statistic Processing Anti-cell proliferative activity was measured four times, and standard deviations were calculated. The significant differences were calculated using Tukey's-test.

Estimation of the Amount of cSBL or Mutant cSBL In-
Table 1. Primers Used in This Study

\begin{tabular}{ll}
\hline \multicolumn{2}{l}{ Primers used for the isolation of cSBL cDNA } \\
AF-1F & TCCTTGTCTCAGAACTGGGCAACATTT \\
AF-112R & CTAAGGACATCGTCCTATTCCAGCAAAA
\end{tabular}

Primers for the introduction of restriction enzyme sites

5'-Nco I site (RC5NcoI) CCATGGCTCAGAACTGGGCAACATTTCCAGC

3'-Bam HI site (RC3BamHI) GGATCCCTAAGGACATCGTCCTATTCAGC

$\begin{array}{cc}\text { Primers for site directed mutagenesis } \\ \text { D24NF } & \text { CAACTGTAACACCATCATGAACAACAATAT } \\ \text { D24KF } & \text { TAACACCATCATGAAGAACAATA } \\ \text { D24RF } & \text { ACCATCATGCGAAACAATA } \\ \text { E88QR } & \text { ACACATATGTAATTAGTTTGCGTTCTGGAG } \\ \text { E88RR } & \text { ACACATATGTAATTAGTTCTCGTTCTGGAG } \\ \text { E97QR } & \text { GGATCCCTAAGGACATCGTCCTATTCCAGCAAA- } \\ & \text { ATGTACGGGATATTGATTCTGACATTT } \\ \text { E97RR } & \text { GCCGGATCCCTAAGGACATCGTCCTATTCCAGCAAA- } \\ & \text { ATGTACGGGATATTGATTCCTACATTTACA }\end{array}$

* Mutated sites are underlined.

ternalized into Tumor Cells The amount of native and mutant cSBL in P388 cells was estimated using RNase activity as a marker. One milliliter of the P388 cell suspension containing $2 \times 10^{5}$ cells was incubated with $2 \mu \mathrm{M}$ cSBL or mutant cSBL in RPMI1640 medium with fetal calf serum for $30 \mathrm{~min}$ at $37^{\circ} \mathrm{C}$. The cells were collected by centrifugation at $200 \times \boldsymbol{g}$ and then washed with phosphate buffered saline (PBS) twice. The cell pellet was suspended in $0.5 \%$ Triton $\mathrm{X}-100$ in $0.1 \mathrm{M}$ Tris- $\mathrm{HCl}$ buffer $(\mathrm{pH} 7.5)$ and frozen and thawed twice, then centrifuged again. The RNase activity of the supernatant was measured, and the amount of enzyme incorporated was calculated on the basis of the specific activity of the cSBL used.

Cloning of cDNA Encoding cSBL The total RNA of bullfrog liver was extracted with TRIZOL reagent (Gibco BBL, Gaithersburg, MD, U.S.A.). ${ }^{14)}$ cSBL-encoded cDNA was obtained from the total RNA by PCR with AF-1F and AF-112R (Table 1) as primers designed according to the cDNA sequence of cSBL reported by Huang et al. ${ }^{15)}$ The cDNA encoding bullfrog cSBL was ligated to pGEM-T easy vector (Promega, WI, U.S.A.). DNA sequences were determined by the method of Sanger and Coulson ${ }^{16)}$ with DNA sequencers LI-COR GENE READER 4200 (LI-COR, NE) and ABI Prism model 377 (Applied Biosystems Japan, Tokyo, Japan). The nucleotide sequence agreed well with that reported by Huang et al. ${ }^{15)}$

Preparation of Site-Directed Mutants of cSBL at the Three Acidic Amino Acid Residues Two primers, RC5NcoI and RC3BamHI, were prepared (Table 1). Nco I and Bam HI sites were added at the $5^{\prime}$ - and $3^{\prime}$-terminus, respectively. The cDNA of cSBL which had restriction sites was amplified with RC5NcoI and RC3BamHI. E97 mutants were prepared by PCR with RC5NcoI, and one of the oligonucleotides, E97QR or E97RR, as primers. The mutants modified at the other sites, E88 and D24, were prepared according to a modified method (Itagaki, T. and Ogawa, Y., unpublished) which changed the method of Landt et al. ${ }^{17)}$ Following PCR amplification between RC5NcoI and E88QR (or E88RR), the product that contained a mutant sequence at E88 was mixed with the cDNA of cSBL at a ratio of $10: 1$. 
Table 2. RNase Activity toward RNA of cSBL and Mutants

\begin{tabular}{lcc}
\hline \hline Mutant & $\begin{array}{c}\text { Specific activity } \\
\text { (units/mg) }\end{array}$ & $\begin{array}{c}\text { Relative activity } \\
(\%)\end{array}$ \\
\hline cSBL & 756 & 100 \\
D24N & 628 & 83.1 \\
E88Q & 336 & 44.4 \\
E97Q & 348 & 46.0 \\
D24R & 214 & 28.3 \\
D24K & 323 & 42.7 \\
E88R & 176 & 23.3 \\
E97R & 91 & 12.0 \\
D24RE88R & 98 & 13.0 \\
D24RE97R & 43 & 5.7 \\
\hline
\end{tabular}

Taq polymerase and dNTP were added to the mixture and reacted at $94^{\circ} \mathrm{C}$ and $72^{\circ} \mathrm{C}$ (20 cycles). The mutated oligonucleotide containing E88Q (or E88R) was annealed with full lenth cSBL cDNA as a template and extended by this reaction. The extended nucleotide was further amplified by PCR. A clone that matched the desired sequence was selected. Similarly, D24 mutants of cSBL were obtained from the PCR products of RC3BamHI and D24NF (D24KF, D24RF). In the case of the double mutants, D24RE88R and D24RE97R, preparations started from the D24R mutant cDNA. Thus we obtained cDNA encoding site-directed mutations at acidic amino acid residues of cSBL having restriction enzyme sites for Nco I and Bam HI at the 5' - and 3 '-terminal, respectively. The mutated cDNA was ligated to an expression vector, and the nucleotide sequence was confirmed by a LI-COR DNA sequencer.

Expression of Site-Directed Mutants from Escherichia coli The cDNA, having been mutated, was ligated to the expression vector pET-pel-RC constructed according to Hang et al. ${ }^{15)}$ from pET22b (Novagene) and transformed into $E$. coli BL21 (DE3) pLysE (Novagene). The cells were cultured with terrific broth at $25^{\circ} \mathrm{C}$ with the addition of $100 \mu \mathrm{g} / \mathrm{ml}$ of ampicillin. The supernatant of $7 \mathrm{~d}$ culture was used for further purification.

Purification of Site-Directed Mutants of cSBL Culture medium (31) was centrifuged at $8000 \mathrm{rpm}$ for $10 \mathrm{~min}$. The supernatant was dialyzed with dialysis tubing (molecular weight 1000 cut) obtained from Spectrapore (Houston, TX, U.S.A.) overnight against de-ionized water. The dialyzate was adjusted to $\mathrm{pH} 6.0$ then applied to a column of P11 $(4 \times 20 \mathrm{~cm})$ equilibrated with $50 \mathrm{~mm}$ sodium phosphate buffer (pH 6.0) and eluted with 11 of the same buffer with a linear gradient of $\mathrm{NaCl}(0-1 \mathrm{M})$. The RNase active fractions were pooled and dialyzed against $10 \mathrm{~mm}$ sodium phosphate buffer ( $\mathrm{pH}$ 6.0). The dialyzed enzyme was applied to a column of Heparin Sepharose CL6B $(1.5 \times 10 \mathrm{~cm})$ equilibrated with $50 \mathrm{~mm}$ sodium phosphate buffer ( $\mathrm{pH} \mathrm{6.0)}$, and eluted with a linear gradient of $\mathrm{NaCl}(0-1 \mathrm{M} \mathrm{NaCl})$ in $200 \mathrm{ml}$ of the buffer. The active fraction was further purified by fast protein liquid chromatography (FPLC) on Mono S 5/5 with a linear gradient of $\mathrm{NaCl}(0-0.3 \mathrm{M})$ in $50 \mathrm{~mm}$ acetate buffer ( $\mathrm{pH} 5.5)$. The purity of the RNase obtained was verified by sodium dodecyl sulfate-polyacrylamide gel electrophoresis (SDSPAGE). From 0.1 to $1 \mathrm{mg}$ of mutant was purified from 31 of culture. CD spectra were recorded for all the cSBL mutants prepared to confirm that they were essentially similar to the

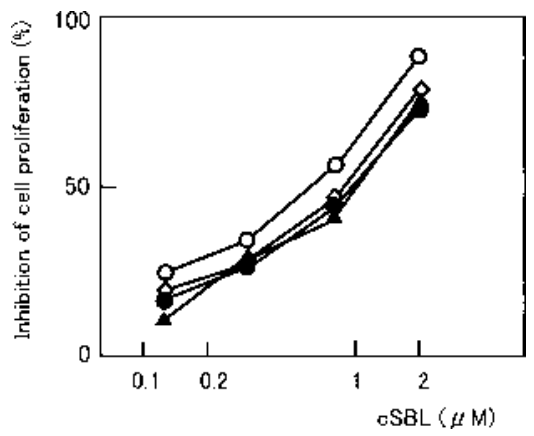

Fig. 2. Inhibition of P388 Cell Proliferation by Native cSBL, D24N, E88Q and E97Q mutant cSBLs

Cells were treated with a given concentration of cSBL and its mutants for $48 \mathrm{~h}$. Cell proliferation without cSBL was normalized to $100 \%$ ( $0 \%$ inhibition). Symbols are $\bullet$, cSBL; O, D24N; $\diamond, \mathrm{E} 88 \mathrm{Q} ; \boldsymbol{\Lambda}, \mathrm{E} 97 \mathrm{Q}$.

native cSBL. The RNase activities of the mutants are shown in Table 2.

Detection of cSBL/cSBL Mutants in Tumor Cells by Fluorescence Microscopy Human leukemia HL60 cells were cultured in RPMI1640 medium, washed twice with ASF104, and adjusted to a concentration of $2 \times 10^{5}$ cells $/ \mathrm{ml}$. $2 \mu \mathrm{M}$ of cSBL or D24RE88R mutant was added, and the cells were cultured further under $5 \% \mathrm{CO}_{2}$ at $37^{\circ} \mathrm{C}$ for $30 \mathrm{~min}$. The cells were then adsorbed on a slide glass pre-treated with poly L-lysine on ice. After fixation with methanol, they were treated with an mouse $\operatorname{IgG}$ monoclonal antibody against cSBL, E86 (prepared by Iwama, M., Sawada, J. et al., unpublished), then FITC (fluorescein)-conjugated goat antibody to mouse IgG $\left(\mathrm{Fab}^{\prime}\right)_{2}(\mathrm{ICN})$, and Vecta shield mounting media with propidium iodide (PI) (Vector Lab, CA, U.S.A.) with $3 \%$ BSA (Sigma fraction V) in PBS containing $20 \mu \mathrm{g} / \mathrm{ml}$ RNase A. The cSBL and PI-stained nucleus were observed with a laser scanning confocal microscope, model LSM510 (Carl Zeiss, Germany), at an excitation wavelength of $488 \mathrm{~nm}$ and $543 \mathrm{~nm}$ for FITC and PI, respectively.

\section{RESULTS}

Anti-tumor Activity of Mutants of cSBL We prepared 7 single mutants of cSBL in which acidic amino acid residues were replaced by the corresponding acid amides (asparagines or glutamine), lysine or arginine. The mutants were D24N, E88Q, E97Q, D24K, D24R, E88R and E97R, and the anti-tumor activities of the mutants toward P388 cells were compared with that of native cSBL. In the former three and the latter four mutants, the positive net charge increased by one and two, respectively, relative to cSBL.

Among acid amide mutants, the D24N mutant slightly increased the inhibition activity of cell proliferation in comparison to native cSBL and the other single mutants (Fig. 2). Since the toxicity of the D24K and D24R mutants was comparable, we decided to study only the arginine mutants (Fig. 3). The anti-tumor activity of the D24R and E88R mutants was markedly increased compared to that of the E97R mutant and native cSBL (Fig. 4). These results suggested that the increase in positive charge at D24 and E88 affected the cytotoxicity of cSBL more than that at E97. Therefore, we prepared two double mutants, D24RE88R and D24RE97R. The increase in the cytotoxicity of D24RE97R was not so 


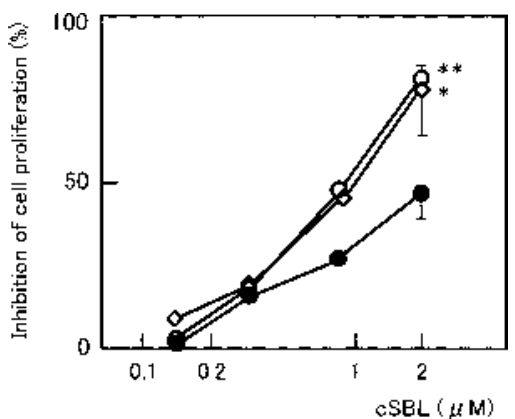

Fig. 3. Inhibition of P388 Cell Proliferation by Native cSBL, D24R and D24K Mutant cSBLs

Cells were treated with a given concentration of cSBL and its mutants for $48 \mathrm{~h}$. Cell proliferation without cSBL was normalized to $100 \%(0 \%$ inhibition). Symbols are cSBL; $\bigcirc, \mathrm{D} 24 \mathrm{R} ; \diamond, \mathrm{D} 24 \mathrm{~K}$. The bar represents the standard deviation. * and **; significantly different from the value of the native cSBL $(p<0.05$ and $p<0.01$, respectively).

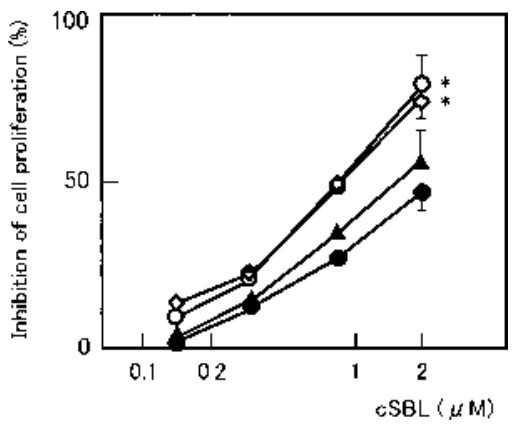

Fig. 4. Inhibition of P388 Cell Proliferation by Native cSBL, D24R, E88R and E97R mutant cSBLs

Cells were treated with a given concentration of cSBL and its mutants for $48 \mathrm{~h}$. Cell proliferation without cSBL was normalized to $100 \%(0 \%$ inhibition). Symbols are cSBL; O, D24R; $\diamond$, E88R; $\boldsymbol{\Delta}$, E97R. The bar represents the standard deviation. $* p<0.05$ compared with the value of the native cSBL.

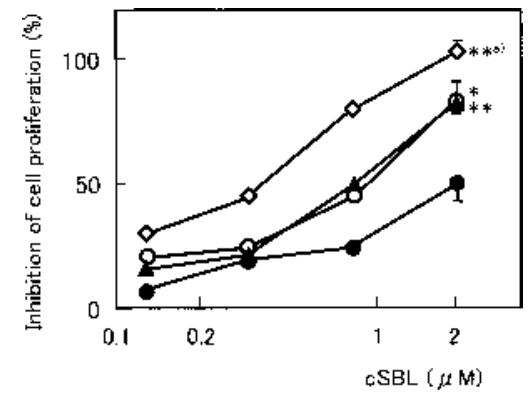

Fig. 5. Inhibition of P388 Cell Proliferation by Native cSBL, D24R, D24RE88R and D24RE97R Mutant cSBLs

Cells were treated with a given concentration of cSBL and its mutan for $48 \mathrm{~h}$. Cel proliferation without cSBL was normalized to $100 \%(0 \%$ inhibition). Symbols are cSBL; O, D24R; $\diamond$, D24RE88R; $\boldsymbol{\Delta}$, D24RE97R. The bar represents the standard deviation. * and **; significantly different from the value of the native cSBL $(p<0.05$ and $p<0.01$, respectively). a) The value was significantly different from the value of D24R or D24RE97R $(p<0.01)$

marked. D24RE88R showed the most remarkable activity among the mutants tested in this study, and its inhibitory action was comparable to the most effective chemically modified cSBL, EDC-ED cSBL, tested so far (Fig. 5).

These results suggest that the substitution of an acidic amino acid residue with arginine increases cytotoxicity in general, and is qualitatively consistent with the finding that an increase in the net charge of cSBL increases cytotoxicity. ${ }^{12)}$ However, the substitution of E97 with arginine was not
Table 3. Internalization of cSBL and Mutants in P388 Cells

\begin{tabular}{lcc}
\hline \hline Mutant & $\begin{array}{c}\text { RNase activity } \\
\text { in the cell (\%) }\end{array}$ & $\begin{array}{c}\text { Mutant } \\
\text { in the cell (\%) }\end{array}$ \\
\hline cSBL & 100 & 100 \\
D24N & 104 & 125 \\
E88Q & 60.2 & 135 \\
E97Q & 50.1 & 109 \\
D24R & 92.9 & 328 \\
E88R & 50.0 & 215 \\
E97R & 35.7 & 298 \\
D24RE88R & 90.5 & 696 \\
D24RE97R & 38.1 & 674 \\
\hline
\end{tabular}

as effective as that of the other acidic acid residues, D24 and E88.

Effect of Mutations on Internalization Efficiency In the previous report, ${ }^{12)}$ we reported that a chemically modified form of cSBL with a higher positive net charge was internalized more effectively into P388 cells than the native cSBL. Therefore, we investigated the relative rate of internalization of the mutant cSBLs. The results are shown in Table 3. Although the mutant cSBLs with an acid amide are internalized into P388 cells at the same rate or slightly higher than the native cSBL, the arginine mutants with an increased net charge are internalized with high efficiency. Double mutants are internalized to an even greater extent, about $6-7$ fold that of the native cSBL. These results are qualitatively similar to those of previous experiments on EDC-modified cSBL in the presence of ethylenediamine. ${ }^{12)}$ Thus, the cytotoxicity seems to be correlated with the efficiency of internalization into cells. Figure 6 shows that there is some correlation between the $\mathrm{IC}_{50}$ value and the amount of $\mathrm{cSBL}$ in the cells under the experimental conditions used, but there is no clear correlation between the $\mathrm{IC}_{50}$ and the total enzymatic activity in the cells.

Detection of the Internalized cSBL/Mutants of cSBL in Human Leukemia HL60 Cells In the previous section, the internalization of cSBL/mutant cSBL into P388 cells was demonstrated using RNase activity as a marker. To confirm the results and visualize the internalized cSBL/mutant cSBL, by fluorescence microscopy, we used human leukemia HL60 cells, because they are larger than, but as sensitive to, cSBL as P388 cells (the $\mathrm{IC}_{50}$ for cSBL and D24RE88R is $1.2 \mu \mathrm{M}$ and $0.15 \mu \mathrm{M}$, respectively). The internalized $\mathrm{cSBL} / \mathrm{mutant}$ cSBL was detected using E86 mouse IgG monoclonal antibody against cSBL, and the FITC-labeled goat antibody for mouse $\operatorname{IgG}$ as the primary and secondary antibodies, respectively. The results are shown in Fig. 7. The fluorescence dye was clearly observed to be bound to cSBL by laser scanning confocal fluorescence microscopy. cSBL and D24RE88R existed in the cytoplasm, and there were clearly more mutant molecules than with native $\mathrm{CSBL}$.

Enzymatic Activities of the Mutants of cSBL (i) Enzymatic Activity toward RNA: Among the cSBL mutants with an acidic amino acid residue replaced by a corresponding amide, the D24N mutant retained about $80 \%$ of the activity of cSBL (Table 2). The activity of the other two was about half that of the native cSBL. However, the activity of the D24R and E88R mutants decreased to about 25\%, and that of the E97R mutant was only $10 \%$ of the activity of the native 

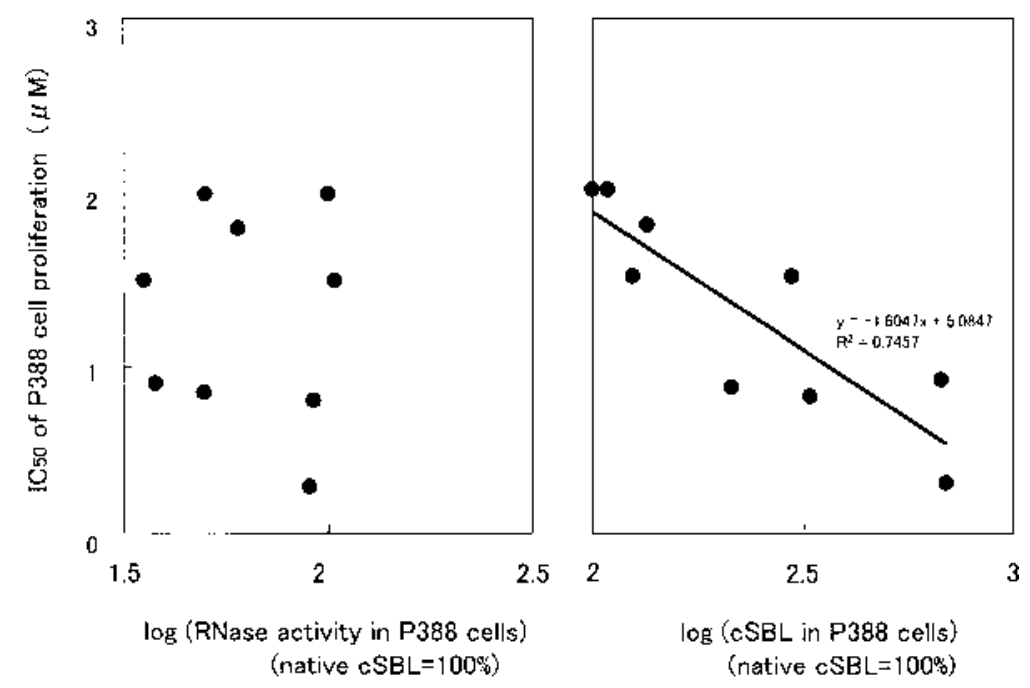

Fig. 6. Correlation between Log Relative Inhibition of Cell Proliferation $\left(\mathrm{IC}_{50}\right)$ and (a) RNase Activity and (b) Amount of cSBL/cSBL Mutants Incorporated into Cells
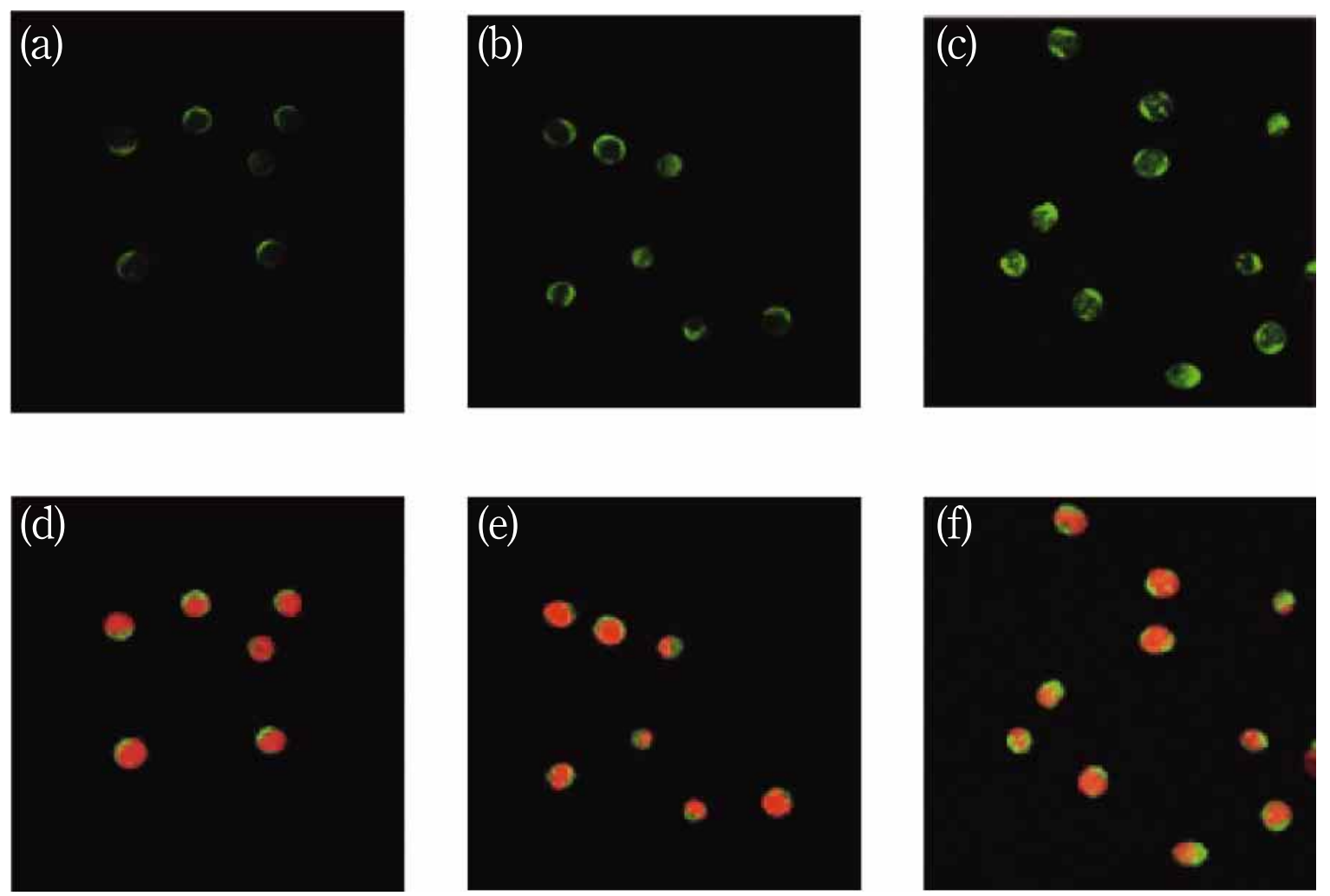

Fig. 7. Microscopic Observation of the Internalization of cSBL and its D24RE88R Mutant into Human HL60 Cells

(a), (d) Control, without cSBL/cSBL mutant treatment. (b), (e) Native cSBL treated cells. (c), (f) D24RE88R mutant-treated cells. (a)—(c) Stained only cSBL. (d)—(f) Stained cSBL and nucleus. Details are described in "Materials and Methods."

cSBL. These results indicated that the effect of substituting a carboxylic amino acid with the corresponding amide on the inactivation of the RNase activity is less than that of an arginine substitution. The decrease in RNase activity of the double mutant D24RE97R toward RNA was most marked, and only $6 \%$ of the activity of cSBL retained. These results seem to suggest that the replacement of E97 with arginine greatly affects the RNase activity against RNA.

(ii) RNase Activity toward Poly U Hybridized with Poly A: In the previous report, ${ }^{13)}$ we observed an increase in the
RNase activity against hybridized RNA on the introduction of basic groups at carboxylic acid residues. Therefore, we here examined the effect of mutating carboxyl groups on double stranded RNA. The results are shown in Fig. 8. The activity to hydrolyze double stranded RNA increased upon the introduction of an arginine residue, especially in the double mutant cSBL, which was about 4-fold as active as the native cSBL. 


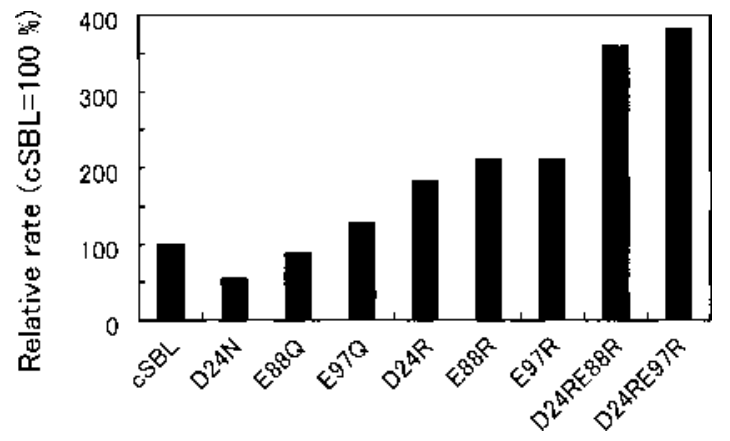

Fig. 8. Relative Hydrolyzing Activity toward Double-Stranded Poly $\mathrm{U} \cdot$ Poly A of cSBL and Site-Directed Mutants of cSBL

\section{DISCUSSION}

The substitution of acidic amino acid residues of cSBL with the corresponding amide or arginine (thereby increasing the net charge) increased the toxicity of cSBL toward P388 cells and HL60 cells, as well as the uptake of cSBL into the tumor cells, and strengthened the RNase activity against double stranded RNA. The results of this report were qualitatively consistent with similar experiments in which the acidic amino acid residues of cSBL were modified chemically with EDC-ethylenediamine. ${ }^{12,13)}$ The substitutions of E97 with arginine and glutamine most affected the enzymatic activities of cSBL, when RNA was the substrate. This may be due to the fact that E97 is one of the constituents of the B2 base recognition site of the RNase A superfamily, and is conserved in the RNase A superfamily, although we do not know the exact location of this group in the substrate analoguecSBL complex.

During the preparation of this paper, Futami et al. reported similar lines of experiments using RNase $\mathrm{A}$ and human RNase 1 and 3T3 SV-40 cells. ${ }^{18)}$ They demonstrated that an increase in the cationization of acidic groups increases cytotoxicity, and the cationized RNase was easily internalized into $3 \mathrm{~T} 3 \mathrm{SV}-40$ cells. In our previous study, we prepared highly toxic derivatives of $\mathrm{cSBL}$ with an $\mathrm{IC}_{50}$ value of about $0.12 \mu \mathrm{M}$ toward P388 cells by chemically modifying the native form in the presence of ethylenediamine. However, it was a mixture of cSBL that was modified, at two of four carboxylic acids. In contrast with these two reports, in the present study, one or two specific residues were substituted with arginine. The most active cSBL mutant, D24RE88R, had a similar potency $\left(\mathrm{IC}_{50}, 0.3 \mu \mathrm{M}\right)$ to the EDC-ED cSBL and was well characterized. Therefore, it might be useful for elucidating the mechanism of cSBL toxicity. However, the $\mathrm{IC}_{50}$ of D24RE88R is slightly larger than that of EDC-ED cSBL. In cSBL, there is another carboxyl group not discussed here. It is a C-terminal proline. The results might suggest that it is necessary to investigate the contribution of this Pro to the cytotoxicity.
The results shown in Fig. 6 suggest the importance of efficient internalizaton into cells to the cytotoxicity of cSBL. The conclusion is the same as that of a previous report. ${ }^{12}$ ) However, the results in Table 3 and Fig. 5 show that the degree of internalization of the two double mutants, D24RE88R and D24RE97R, was very similar but the cytotoxcity of D24RE88R was markedly higher than that of D24RE97R. This suggests that the internalization is one, but not the only, important factor for cytotoxicity. Therefore, to find the other factors influencing cytotoxicity, it might be necessary to study how the change in the enzymatic properties of the cSBL mutants and the chemically modified cSBLs contributes to cytotoxicity more closely. A similar change in enzymatic properties, such as an increase in RNase activity against double stranded RNA and some change in B2 site specificity of cSBL, were observed in both cases. Mutant cSBLs may prove a more potent cytotoxin in cells, acting to hydrolyze hybridized RNA or other specific sites of RNA.

\section{REFERENCES AND NOTES}

1) Present address: Department of Materials Engineering, Nagaoka National College of Technology, 888 Nishikatakai, Nagaoka, Niigata 940-8532, Japan.

2) Nitta K., Takayanagi G., Kawauchi H., Hakomori S., Cancer Res., 47, $4877-4883$ (1987).

3) Nitta K., Oyama F., Oyama R., Sekiguchi K., Kawauchi H., Takayanagi Y., Hakomori S., Titani K., Glycobiol., 3, 37-45 (1993).

4) Smyth D. G., Stein W. H., Moore S., J. Biol. Chem., 238, 227-234 (1963).

5) Irie M., Nitta K., Nonaka T., Cell Mol. Life Sci., 54, 775-784 (1998).

6) Okabe Y., Katayana N., Iwama M., Watanabe H., Ohgi K., Irie M., Nitta K., Kawauchi H., Takayanagi Y., Oyama F., Titani K., Abe Y., Okazaki T., Inokuchi N., Koyama T., J. Biochem. (Tokyo), 109, 786790 (1991).

7) Nitta R., Katayama N., Okabe Y., Iwama M., Watanabe H., Abe Y., Okazaki T., Ohgi K., Irie M., J. Biochem. (Tokyo), 106, 729-735 (1989).

8) Ardelt W., Mikulski S. M., Shogen K., J. Biol. Chem., 266, 245-251 (1991).

9) Kamiya Y., Oyama F., Oyama R., Sakakibara F., Nitta K., Kawauchi H., Takayanagi Y., Titani K., J. Biochem. (Tokyo), 108, 139-143 (1990).

10) Titani K., Takio K., Kuwada M., Nitta K., Sakakibara F., Kawauchi H., Takayanagi G., Hakomori S., Biochemistry, 26, 2189-2194 (1987).

11) Youle R. J., D'Alessio G., "Ribonucleases: Structures and Functions," ed. by D’Alessio G., Riordan J. F., Academic Press, New York, 1997, pp. $491-514$.

12) Iwama M., Ogawa Y., Sasaki N., Nitta K., Takayangi Y., Ohgi K., Tsuji T., Irie M., Biol. Pharm. Bull., 24, 978-981 (2001).

13) Iwama M., Ogawa Y., Ohgi K., Tsuji T., Irie M., Biol. Pharm. Bull., 24, 1366-1369 (2001).

14) Chomczynski P., Sacchi N., Anal. Biochem., 162, 156-159 (1987).

15) Huang H. C., Wang S. C., Leu I. Y., Leu S. C., Liao Y. D., J. Biol. Chem., 273, 6395-6401 (1998).

16) Sanger F., Coulson A. R., J. Mol. Biol., 94, 441- 448 (1975).

17) Landt O., Grunert H. P., Hahn U., Gene, 96, 125-128 (1990).

18) Futami J., Maeda T., Kitazoe M., Nukui E., Tada H., Seno M., Kosaka M., Yamada H., Biochemistry, 40, 7518-7524 (2001). 